\title{
Análise de programas escolares de saúde bucal no Brasil
}

\author{
Analysis of oral health programs in Brazil
}

\author{
Débora Santos Sityá* \\ Giuliano Omizzolo Giacomini* \\ Luis Antônio Sangioni*** \\ Carla da Rocha Sartori Sendtko ${ }^{* * * *}$ \\ Beatriz Unfer ${ }^{* * * *}$
}

\section{Resumo}

Os programas de saúde bucal com ações preventivas, educativas e/ou cirúrgico-restauradoras contribuem para aumentar a qualidade de vida da população. No meio escolar, o ambiente e a faixa etária dos escolares favorecem o aprendizado e a motivação. Objetivo: analisar as atividades de saúde bucal realizadas em escolas brasileiras durante o período de 2002 a 2012. Revisão de literatura: os artigos foram pesquisados na base de dados da Bibliografia Brasileira de Odontologia (BBO) com a utilização das expressões: "saúde bucal escolar", "programa escolar", "assistência odontológica escolar", "educação em saúde bucal escolar", "higiene bucal escolar", "promoção de saúde bucal escolar" e "escovação dentária escolar". Os resultados mostram variação nas modalidades de ações empregadas, com predominância dos componentes educativo e preventivo, e utilização de recursos audiovisuais, demonstrações práticas de escovação e escovação supervisionada. Considerações finais: conclui-se que os programas escolares implantados no Brasil são tradicionais, sem a introdução de estratégias inovadoras nas abordagens educativas e que deveriam utilizar instrumentos de avaliação mais efetivos para monitorar os efeitos das ações de saúde dirigidas à comunidade escolar.

Palavras-chave: Saúde bucal. Saúde escolar. Avaliação de programa.

\section{Introdução}

A saúde bucal deve ser vista como parte integrante e indissociável da saúde geral do indivíduo. Os programas de saúde bucal com ações preventivas, educativas e/ou cirúrgico-restauradoras são fundamentais, não apenas para melhorar as condições da saúde bucal, mas também para aumentar a qualidade de vida da população.

A idade escolar é um período adequado para o desenvolvimento de programas de saúde. Os escolares apresentam maior facilidade de aprendizagem, melhor coordenação motora e maior motivação para incorporar hábitos saudáveis ${ }^{1,2}$. Com base nisso, programas escolares de saúde bucal poderão gerar maior responsabilidade e preocupação com a higiene oral, reduzindo a necessidade de tratamentos invasivos ao longo da vida e, consequentemente, desenvolvendo hábitos de vida mais saudáveis.

Tendo em vista a importância dos programas escolares de saúde bucal como forma de atuação na área educativa, preventiva e de recuperação da saúde, realizou-se uma revisão de literatura com o objetivo de avaliar os programas de saúde bucal executados no Brasil durante o período de 2002 a 2012.

Acadêmica do curso de Odontologia da Universidade Federal de Santa Maria (UFSM); Monitora Bolsista do Programa de Educação pelo Trabalho para a Saúde (PET-Saúde), Santa Maria, RS, Brasil.

Acadêmico curso de Odontologia da Universidade Federal de Santa Maria (UFSM); Monitor Bolsista do Programa de Educação pelo Trabalho para a Saúde (PET-Saúde), Santa Maria, RS, Brasil.

Professor Doutor associado do Departamento de Medicina Veterinária Preventiva Centro de Ciências Rurais da Universidade Federal de Santa Maria (UFSM); tutor do Programa de Educação pelo Trabalho para a Saúde (PET-Saúde), Santa Maria, RS, Brasil.

**** Cirurgiã-dentista; Especialista em Saúde Coletiva pelo Centro Universitário Franciscano (Unifra), Prefeitura Municipal de Santa Maria, Secretaria Municipal da Saúde, Superintendência de Vigilância em Saúde; preceptora do Programa de Educação pelo Trabalho para a Saúde (PET-Saúde), Santa Maria, RS, Brasil.

**** Professora Doutora associada no Departamento de Estomatologia da Universidade Federal de Santa Maria (UFSM); tutora do Programa de Educação pelo Trabalho para a Saúde (PET-Saúde), Santa Maria, RS, Brasil. 
Buscou-se verificar a modalidade desses programas e os tipos de atividades desenvolvidas nas escolas.

\section{Materiais e método}

Foi realizada uma revisão bibliográfica sobre programas escolares de saúde bucal, desenvolvidos no Brasil, no período de 2002 a 2012, em periódicos nacionais. Os artigos foram levantados na base de dados da Bibliografia Brasileira de Odontologia (BBO), com a utilização das expressões: "saúde bucal escolar", "programa escolar", "assistência odontológica escolar", "educação em saúde bucal escolar", "higiene bucal escolar", "promoção de saúde bucal escolar" e "escovação dentária escolar". Do total de 686 referências encontradas, foram excluídos artigos que não abordavam como tema principal os programas escolares, os artigos de revisão, os levantamentos exclusivamente epidemiológicos e aqueles que não puderam ser recuperados com o texto completo.

\section{Resultados e discussão}

A seleção dos artigos resultou em 17 trabalhos para análise neste estudo ${ }^{3-19}$. Os dados obtidos foram organizados em tabelas, de acordo com a modalidade, com o tipo de atividade e com os resultados apresentados pelos programas escolares.

Verificou-se que os programas apresentaram variedade de composição nas modalidades empregadas para a atuação em escolas, com predominância dos componentes educativos e preventivos, presentes em $82 \%$ e $76 \%$ das atividades, respectivamente. Apenas quatro programas tinham o componente clínico como atividade (Tabela 1 ).

Tabela 1 - Número e proporção de artigos publicados em periódicos nacionais sobre programas escolares de saúde bucal, no período de 2002 a 2012, segundo a modalidade empregada. Brasil, 2013

\begin{tabular}{l|r|r}
\hline \multicolumn{1}{|c|}{ MODALIDADE } & \multicolumn{1}{c|}{ № } & \multicolumn{1}{c}{$\%$} \\
\hline Educativo - preventivo $^{5,12,13,16}$ & 4 & 23 \\
Educativo $^{4,7,14}$ & 3 & 17 \\
Educativo - preventivo - epidemiológico $^{9,17,18}$ & 3 & 17 \\
Educativo - preventivo - epidemiológico - clínico $^{8,15,19}$ & 3 & 17 \\
Epidemiológico - preventivo $^{6,10}$ & 2 & 12 \\
Preventivo - epidemiológico - clínico $^{11}$ & 1 & 7 \\
Epidemiológico - educativo $^{3}$ & 1 & 7 \\
Total & 17 & 100 \\
\hline Fonte: dos autores. &
\end{tabular}

Fonte: dos autores.

Os artigos analisados apresentam-se sobrescritos em cada item da tabela.

Embora se deva considerar a capacidade técnica para a execução de cada programa implementado, é preciso chamar a atenção para o fato de que atividade de saúde isolada do contexto da integralidade da atenção em saúde tem a probabilidade de gerar pouco ou nenhum impacto na população alvo. O uso da terminologia promoção de saúde bucal, frequentemente encontrada nos artigos pesquisados, remete somente a atividades educativas e preventivas de saúde bucal como, por exemplo, atividades audiovisuais, escovação supervisionada e aplicação de flúor. Todavia, o termo tem um conceito mais amplo, com origem na promoção de saúde discutida no contexto nacional e internacional. Conforme a Carta de Ottawa (1986), documento chave das discussões sobre $o$ tema para promover saúde, é necessário haver paz, educação, moradia, alimentação, renda, ecossistema estável, justiça social e equidade ${ }^{20}$. Para tanto, é necessário capacitar a comunidade para atuar e controlar a melhoria de sua qualidade de vida e saúde. Nesse contexto, a promoção da saúde bucal deveria estar inserida em um conceito amplo de saúde que transcende a dimensão meramente técnica e específica do setor odontológico, integrando a saúde bucal às demais práticas de saúde coletiva ${ }^{20}$. Essas premissas que deveriam nortear as ações de saúde bucal não são colocadas em discussão nos artigos analisados.

A descrição das atividades realizadas de acordo com a modalidade empregada nos programas analisados (Tabela 2) mostra que a utilização de recursos audiovisuais, a escovação supervisionada, a verificação de índices periodontais e o uso do tratamento restaurador traumático foram mais frequentes.

Tabela 2 - Número e proporção das atividades, segundo cada modalidade de programa escolar de saúde bucal, relatadas em artigos de periódicos nacionais, no período de 2002 a 2012. Brasil, 2013

\begin{tabular}{|c|c|c|c|}
\hline Modalidade & Atividade & N. $\stackrel{\circ}{ }$ & $\%$ \\
\hline \multirow{7}{*}{ Educativa } & Audiovisual| $4,5,7-9,12,13,15-17$ & 10 & 26 \\
\hline & Demonstrações práticas ${ }^{4,5,8,9,15-18}$ & 8 & 21 \\
\hline & Atividades lúdicas $4,5,8,12,13,15$ & 6 & 16 \\
\hline & Palestras ${ }^{3,8,12,16-18}$ & 6 & 16 \\
\hline & Material escrito $4,5,8,16,18$ & 5 & 13 \\
\hline & Outros $^{5,14,15}$ & 3 & 8 \\
\hline & Total & 38 & 100 \\
\hline \multirow{6}{*}{ Preventiva } & Escovação supervisionada ${ }^{6,5,8-12,15-18}$ & 11 & 42 \\
\hline & Aplicação de flúor ${ }^{8,10,11,15-17,19}$ & 7 & 27 \\
\hline & Revelação de placa ${ }^{6,5,8,9,17,18}$ & 6 & 23 \\
\hline & Remoção profissional de placa ${ }^{9}$ & 1 & 4 \\
\hline & Cariostático ${ }^{11}$ & 1 & 4 \\
\hline & Total & 26 & 100 \\
\hline \multirow{3}{*}{ Epidemiológica } & Índices periodontais ${ }^{3,6,9-11,15,17,18}$ & 8 & \\
\hline & Índices de cárie ${ }^{6,8,10,15,19}$ & 5 & \\
\hline & Total & 13 & 100 \\
\hline \multirow{3}{*}{ Clínica } & ART $^{11,15,19}$ & 3 & 75 \\
\hline & Cirúrgico-restaurador $^{8}$ & 1 & 25 \\
\hline & Total & 4 & 100 \\
\hline
\end{tabular}

Fonte: dos autores.

Os artigos analisados apresentam-se sobrescritos em cada item da tabela. 
Chama a atenção que as formas de aprendizagem ativa, como atividades práticas e lúdicas ainda competem em desvantagem com metodologias nas quaiso sujeito é passivo em relação ao seu aprendizado. A utilização de recursos de educação colocados, de forma abstrata, às pessoas torna difícil sua contextualização na realidade cotidiana ${ }^{21}$. As estratégias tradicionais e de ênfase na determinação do comportamento ideal não possibilitam o diálogo nem a participação das pessoas envolvidas. A construção do conhecimento, com os envolvidos, possibilitaria maior autonomia na decisão de cuidados de saúde bucal $^{21}$. Embora a educação em saúde, realizada no ambiente escolar, favoreça o envolvimento da criança para construir novos conhecimentos, neste estudo, constatou-se que poucos programas incluíram os professores e os pais nas atividades desenvolvidas. Os professores, por disporem de conhecimentos pedagógicos e psicológicos, facilitariam a mudança de atitudes, hábitos e cuidados. $\mathrm{E}$ a participação dos pais ou responsáveis conduziria para que os ensinamentos repassados às crianças fossem incorporados a suas rotinas e reforçados ao longo do tempo, em casa, em uma motivação continuada ${ }^{2}$. A construção do conhecimento por todas as pessoas envolvidas no processo possibilitaria maior autonomia na tomada de decisão para os cuidados de saúde bucal ${ }^{20,21}$.

A escolha da técnica de aplicação de flúor deve ocorrer depois de constatada a necessidade em função do risco de cárie e da avaliação dos custos e aspectos operacionais, o que não foi especificado nos artigos estudados. É sabido que o flúor pode ser utilizado, de forma coletiva ou individual, para a prevenção de cárie dentária. Contudo, a associação de método sistêmico (flúor na água de abastecimento público) com métodos tópicos deveria ter justificativa epidemiológica. Além disso, o uso de um método tópico de aplicação de flúor, associado ao uso de dentifrício fluoretado, é recomendado quando há ausência de água fluoretada, considerando-se os aspectos operacionais e de custos desses métodos ${ }^{22}$. Para cuidados bucais efetivos há a necessidade da construção de políticas públicas saudáveis e o desenvolvimento de estratégias direcionadas a todas as pessoas, garantindo o acesso à água tratada e fluoretada a todos, a universalização do uso de dentifrício fluoretado e escova dental e a disponibilidade de cuidados odontológicos apropriados ${ }^{22}$.

Sobre os resultados alcançados pelos programas, verificou-se que esses trouxeram mudanças de conhecimentos e consciência e de atitudes (alteração de índices, melhora na higiene oral, redução da cárie dental) (Tabela 3). Chama a atenção o fato de que sete programas escolares não provocaram as mudanças desejadas durante o período em que foram implantados.
Tabela 3 - Número e proporção dos resultados alcançados pelos programas escolares relatados em artigos de periódicos nacionais, no período de 2002 a 2012. Brasil, 2013

\begin{tabular}{|c|c|c|}
\hline Resultados & N. $\stackrel{o}{ }$ & $\%$ \\
\hline Conhecimento e consciência ${ }^{3-5,7,9,12,14-19}$ & 12 & 43 \\
\hline Mudança de atitude $e^{4,5,9,13,15-19}$ & 9 & 32 \\
\hline Não promoveu mudanças ${ }^{6,8,10,16}$ & 4 & 14 \\
\hline $\begin{array}{l}\text { Necessidade de prolongar o tempo do } \\
\text { programa }^{6,15,17}\end{array}$ & 3 & 11 \\
\hline Total & 28 & 100 \\
\hline
\end{tabular}

Fonte: dos autores.

Os artigos analisados apresentam-se sobrescritos em cada item da tabela.

A avaliação da eficiência dos programas escolares foi realizada somente ao final dos programas, mas reconhece-se o fato de que deveria ser realizada durante o processo, ponderando-se os impactos dos resultados finais ${ }^{23}$. Portanto, as mudanças detectadas em curto prazo e somente ao final são insuficientes para garantir que as transformações pretendidas tenham surtido efeito e permaneçam na população beneficiada pelos programas. Nesse sentido, torna-se também essencial o levantamento epidemiológico como uma ferramenta para gerar dados para o conhecimento das necessidades de saúde, para o planejamento de ações e, em médio e longo prazo, para mostrar a eficácia dos programas com a diminuição das doenças bucais ${ }^{2,21}$.

Como as limitações do estudo, salienta-se a publicação restrita de estudos sobre programas escolares, embora seja sabido que essas atividades ocorrem frequentemente na maioria dos municípios brasileiros. Uma explicação pode ser justamente o que foi encontrado como resultado neste estudo: muitas das atividades de saúde bucal são dirigidas a escolares como uma tarefa rotineira para professores e estudantes de Odontologia em programas de extensão que não visam a realização de uma pesquisa científica. Outra razão é que os profissionais de saúde bucal, inseridos principalmente na Estratégia de Saúde da Família, não tem como rotina a divulgação científica de sua prática em serviços de saúde.

A opção por utilizar a base de dados da Bibliografia Brasileira de Odontologia foi porque nessa base de dados encontra-se a maioria das publicações brasileiras com a abordagem do tema e no período proposto por este estudo.

\section{Considerações finais}

A partir da análise dos artigos, conclui-se que os programas escolares implantados no Brasil são tradicionais, sem introdução de estratégias inovadoras nas abordagens educativas, os quais deveriam utilizar instrumentos de avaliação efetivos para monitorar os efeitos das ações de saúde dirigidas à comunidade escolar. 


\section{Abstract}

Oral health programs with preventive, educational, and/or surgical-restorative actions contribute to increase the quality of life of the population. In school, the environment and the age group of students promote learning and motivation. Objective: to analyze oral health activities performed in Brazilian schools from 2002 to 2012. Literature review: articles were obtained from the $B B O$ (Brazilian Bibliography of Dentistry) database, with the use of expressions: "school oral health", "school program", "school dentistry services", "school oral health education", "school oral hygiene", "school oral health promotion", and "school teeth brushing". Results show variation in methods of actions applied with the predominance of educational and preventive components, and the use of audiovisual resources, practical presentation of brushing, and supervised brushing. Final considerations: it is concluded that the school programs implemented in Brazil are traditional with no introduction of innovative strategies in educational approaches, also they should use more effective assessment instruments in order to monitor the effects of health actions aimed at the school community.

Keywords: Oral health. School health. Program evaluation.

\section{Referências}

1. Figueira TR, Leite ICG. Percepções, conhecimentos e práticas em saúde bucal de escolares. RGO. 2008; 56(1):27-32.

2. Vasconcelos R, Da Matta ML, Pordeus IA, De Paiva SM. Escola: um espaço importante de informação em saúde bucal para a população infantil. Rev Fac Odontologia São José dos Campos 2011; 4(3): 43-51.

3. Aquilante AG, De Almeida BS, De Castro RFM, Xavier CRG, Peres SHCS, Bastos JRM. A importância da educação em saúde bucal para pré-escolares. Rev de Odontol da Unesp 2003; 32(1):39-45.

4. Conrado CA, Maciel SM, Oliveira MR. Programa educacional em saúde bucal baseado em escolas: a experiência de Maringá-PR, Brasil. J Appl Oral Sci 2004; 12(1):27-33.

5. De Almeida ACP, Zanetti HHV, Peixoto AD, Barros CDR, Septimio DM, Bueno GCM, et al. Promoção de saúde bucal através de orientação, motivação e controle de placa. RBO. 2003; 60(6):387-9.

6. Do Amaral RC, Rihs LB, De Sousa MLR, Wada RS. Avaliação longitudinal de um programa odontológico direcionado a crianças de instituições de ensino infantil de Piracicaba, São Paulo, Brasil. Rev Fac Odontol Univ Passo Fundo 2009; 14(3):203-10.

7. Ferrucio M, Sydney GB, Ferrucio E, Sydney RB. O papel da educação odontológica escolar na manutenção do elemento dental traumatizado. Rev ABO Nac. 2003/2004; 11(6):336-42.

8. Freire MCM, Soares FF, Higino MASP, Nunes MF, Soares EF, Nunes FCS. Experiência de cárie em crianças de instituições de educação infantil com e sem assistência odontológica. Rev Odonto Ciênc. 2009; 24(1):64-70.

9. Leal SC, Bezerra ACB, De Toledo OA. Effectiveness of teaching methods for toothbrushing in preschool children. Braz Dent J 2002; 13(2):133-6.

10. Meneghim MC, Esmeriz CEC, Vilas-Boas P, Meneghim ZMP, Pereira AC. Impacto de programas educativos sobre condições bucais de escolares de 6 e 7 anos em duas escolas municipais do interior do Estado de São Paulo/Brasil. Arq Odontol 2012; 48(1):40-6.

11. Moraes NM, Knupp R, Cabral I. Avaliação de dezoito meses de um programa de saúde bucal em alunos de uma escola municipal do Rio de Janeiro. RBO 2004; 61(3-4):227-8.

12. Orsi VME, Pereira AA, Flório FM, De Souza LZ, Boaretto P, Pinheiro PPS, et al. Hábitos e conhecimentos de escolares sobre saúde bucal. RGO. 2009; 57(3):291-6.

13. Pereira VP, Schardosim LR, Da Costa CT. Remoção do hábito de sucção de chupeta em pré-escolares: apresentação e avaliação de uma estratégia motivacional. Rev Fac Odontol. 2009; 50(3):27-31.

14. Ribeiro DG, Dovigo LN, Da Silva SRC. Avaliação de um método educativo em saúde bucal aplicado em escolares de ensino público. Arq em Odontol. 2009; 45(3):154-9.

15. Rihs LB, De Souza MLR, Wada RD, Gushi LL. Auto-aplicação de gel fluoretado por pré-escolares de Piracicaba, SP. Rev Odontol Univ Cid São Paulo 2005; 17(1):55-62.

16. Siqueira MFG, Jardim MCAM, Sampaio FC, Vasconcelos LCS, Vasconcelos, LC. Evaluation of an oral health program for children in early childhood. Rev Odonto Ciênc 2010; 25(4):350-4.

17. Toassi RFC, Petry PC. Motivação no controle do biofilme dental e sangramento gengival em escolares. Rev Saúde Pública 2002; 36(5):634-7.

18. Valle DD, Vianna RBC, Quintanilha LELP, Neves TF. Avaliação de um Programa de Promoção de Saúde utilizando diferentes indicadores: estudo piloto. Rev ABO Nac. 2003; 11(5):299-303

19. Vaz PRM, Vieira FFR, Silveira RG, Miasato JM. Odontopediatria social: resultados de um ano do projeto Sorria Bairro-Escola nas creches públicas de Nova Iguaçu. Rev Bras Odontol. 2009; 66(2):165-9.

20. Carta De Ottawa - Primeira conferência internacional sobre promoção da saúde, Ottawa, nov.1986.

21. Pauleto ARC, Pereira MLT, Cyrino EG. Saúde bucal: uma revisão crítica sobre programações educativas para escolares. Rev Ciência e Saúde Coletiva 2004; 9(1):121-30.

22. Brasil. Ministério da Saúde. Guia de recomendações para o uso de fluoretos no Brasil. Ministério da Saúde: Brasília, 2009.

23. Center for the advancement of community based public health. Uma estrutura de avaliação para programas de saúde em comunidades. Durham, North Carolina, Jun. 2000.

Endereço para correspondência:

Giuliano Omizzolo Giacomoni

BR 158 - km 323,6/1555 - Bairro Cerrito

97060-090 Santa Maria-RS

Fone: (55) 99575022

(55) 32221229

E-mail: giulianoog@gmail.com

Recebido: 22/04/2014. Aceito: 02/11/2014. 